\title{
Upregulated microRNA-199a-5p inhibits nuclear receptor corepressor 1 translation in mice with non-alcoholic steatohepatitis
}

\author{
BINGGUI ZHANG ${ }^{1}$, RONGQI WANG ${ }^{1}$, JINGHUA DU $^{1}$, JINGYA NIU $^{2}$, RUI ZHANG $^{3}$, \\ SHUNJIANG XU ${ }^{3}$, XUEMIN NIU ${ }^{1}$, QINGFU ZHANG ${ }^{3}$ and YUEMIN NAN ${ }^{1}$ \\ ${ }^{1}$ Department of Traditional and Western Medical Hepatology, The Third Hospital of Hebei Medical University, Shijiazhuang, \\ Hebei 050051; ${ }^{2}$ Department of General Surgery, Hebei Provincial Geriatric Hospital, Shijiazhuang, Hebei 050031; \\ ${ }^{3}$ Central Laboratory, The First Hospital of Hebei Medical University, Shijiazhuang, Hebei 050051, P.R. China
}

Received December 4, 2013; Accepted July 4, 2014

DOI: $10.3892 / \mathrm{mmr} .2014 .2592$

\begin{abstract}
Mounting evidence indicates that dysregulated microRNAs (miRNAs) are important in the etiology and pathogenesis of steatohepatitis. However, the functions of miRNAs in the pathophysiological process of non-alcoholic steatohepatitis (NASH) are poorly understood. In this study, C57BL/6J mice were fed a methionine-choline-deficient (MCD) diet for eight weeks in order to induce hepatic steatohepatitis. Using reverse transcription polymerase chain reaction, the hepatic expression levels of miR-199a-5p, miR-122 and miR-221 in the mice were examined. Bioinformatic analysis of dysregulated miR-199a-5p was performed to predict the potential role of miR-199a-5p in NASH. The MCD diet was found to significantly reduce miR-122 expression levels and significantly increase miR-199a-5p expression levels in mouse livers, compared with those of mice fed a control diet. In the bioinformatic analysis, miR-199a-5p was identified to be predominantly involved in transcription, protein serine/threonine kinase activity, insulin signaling, and the Wnt and mitogen-activated protein kinase signaling pathways. The regulation of nuclear receptor corepressor 1 (NCOR1) by miR-199a-5p was also examined by silencing and overexpressing this miRNA in LX-2 cells. The data revealed that NCOR1 protein levels were significantly reduced and enhanced by miR-199a-5p mimic and inhibitor, respectively. These findings suggest a key role for miR-199a-5p in the progression of NASH through inhibition of NCOR1 translation, and provide novel insights into NASH pathogenesis.
\end{abstract}

Correspondence to: Dr Yuemin Nan, Department of Traditional and Western Medical Hepatology, The Third Hospital of Hebei Medical University, 139 Ziqiang Road, Shijiazhuang, Hebei 050051, P.R. China

E-mail: nanyuemin@163.com

Key words: non-alcoholic steatohepatitis, microRNA-199a-5p, methionine-choline-deficient, bioinformatic analysis, nuclear receptor corepressor 1

\section{Introduction}

Non-alcoholic steatohepatitis (NASH), part of the spectrum of non-alcoholic fatty liver disease (NAFLD), is characterized by insulin resistance and dyslipidemia, and may progress to fibrosis, cirrhosis, liver failure and hepatocellular carcinoma (1). Recently, NASH has been recognized as a leading cause of severe hepatic dysfunction and as a result has attracted increasing attention. The successful prevention and treatment of NASH at an early stage greatly depends on an improved understanding of the underlying molecular mechanisms involved in the disease. A variety of factors, including lipid metabolism dysregulation, insulin resistance, immune response, inflammation and oxidative stress, contribute to the pathogenesis of NASH $(2,3)$.

A methionine-choline-deficient (MCD) diet is a common method used in laboratory animals to induce NASH, causing hepatic steatosis with inflammation, which is morphologically identical to NASH in humans $(4,5)$. Using this model, oxidative stress has been shown to contribute to the progression of NASH by stimulating immune responses, indicating the possible role of adaptive immunity in the pathogenesis of the disease (6). However, the exact cause of steatohepatitis remains poorly understood.

MicroRNAs (miRNAs) are fundamental in diabetes and NAFLD, and correcting miRNA expression through either upregulation or inhibition has been suggested as a promising treatment for metabolic syndrome, and may alleviate disease progression (7). miRNAs are a class of single-stranded non-coding small RNAs, typically 22 nucleotides in length, that regulate protein expression at the posttranscriptional level, via completely or partially binding to protein-coding mRNAs (8). Currently, 2,042 mature human miRNAs have been identified (miRBase 19.0, October 17, 2012), which regulate over a third of human genes (9). miRNAs are associated with numerous pathophysiological events, such as tissue development, differentiation, cell proliferation and apoptosis $(10,11)$.

miR-33a and miR-33b have been reported to be involved in controlling cholesterol and lipid homeostasis by regulating the expression of sterol-regulatory element binding protein 
(SREBP) (12). In addition, miR-375 was found to reduce insulin secretion and affect insulin downstream signaling (13). Notably, knockdown of miR-122, an abundant miRNA specifically expressed in the human liver, was shown to reduce hepatitis $\mathrm{C}$ virus protein replication while inducing heme oxygenase 1 expression (14). Furthermore, upregulation of miRNA-221/222 activated stellate cells and promoted the progression of liver fibrosis (15). The abundance of miR-199a-5p/3p has also been reported to correlate with the progression of liver fibrosis (16).

Notably, circulating miRNAs have been suggested as useful tools in the diagnosis of liver diseases $(17,18)$. Alterations in the expression levels of miR-29c, miR-34a, miR-155 and miR-200b, hepatic miRNAs, were associated with differences in the pathophysiological and pathomorphological features of NASH (19). Furthermore, in a study screening for altered expression levels of hepatic miRNAs associated with NASH, a total of 23 miRNAs were reported to be underexpressed or overexpressed in NASH, with the predicted targets of these miRNAs known to affect cell proliferation, metabolism, protein translation, apoptosis, inflammation and oxidative stress (20).

In the present study, the expression levels of liver miR-199a-5p, miR-122 and miR-221 were examined in a mouse model of NASH using reverse transcription polymerase chain reaction (RT-PCR). The target genes of miR-199a-5p were predicted by bioinformatic tools, including TargetScan 6.2, miRanda and PITA. Nuclear receptor corepressor 1 (NCOR1), with NASH-related functions, emerged as an miR-199a-5p target gene predicted by all algorithms. NCOR1 is a large, multi-domain protein that is recruited by nuclear receptors to mediate transcriptional repression, and has been implicated in the epigenetic regulation of circadian and metabolic physiology through activation of histone deacetylase 3 (21) and other physiological pathways, including cancer promotion (22).

In the present study, NCOR 1 was examined in vitro by silencing and overexpressing miR-199a-5p in LX-2 human hepatic stellate cells (HSCs).

\section{Materials and methods}

Animals, diets and experimental design. Male C57BL/6J mice, aged six weeks, were obtained from the Experimental Animal Center of the Chinese Academy of Medical Sciences (Beijing, China), and housed under a $12 \mathrm{~h}$ light/dark cycle at $24^{\circ} \mathrm{C}$, with free access to purified water and a standard diet. After two weeks acclimation, the mice were randomly divided into two groups, fed either an MCD diet (ICN Biomedicals, Aurora, OH, USA) or a normal diet supplemented with choline bitartate $(2 \mathrm{~g} / \mathrm{kg})$ and DL-methionine $(3 \mathrm{~g} / \mathrm{kg}$; ICN Biomedicals) for eight weeks. Animal food was stored at $4^{\circ} \mathrm{C}$ prior to use and was available ad libitum. At the end of the eight-week exposure, blood samples from retro-orbital veins were collected rapidly for biochemical analysis and then the mice were sacrificed by cervical dislocation. At the end of the eight-week exposure, the mice were sacrificed. The livers were excised, snap-frozen in liquid nitrogen and stored at $-80^{\circ} \mathrm{C}$ until use, or fixed in $10 \%$ formalin for histological analysis. All experimental procedures were approved by the Animal Experimentation Ethics Committee of the Hebei Medical University (Shijiazhuang, China).
Biochemical analysis. Serum alanine aminotransferase (ALT) and aspartate aminotransferase (AST; Shanghai Kehua Bio-Engineering Co., Ltd., Shanghai, China) levels, reliable indicators of liver inflammatory injury, were measured using an Olympus AU5400 automatic chemical analyzer (Olympus Corporation, Tokyo, Japan) according to the manufacturer's instructions.

Histological analysis. Paraffin-embedded liver sections (5 $\mu \mathrm{m})$ were stained with hematoxylin and eosin, and Masson's trichrome to evaluate hepatic steatosis and fibrosis, as described previously $(23,24)$. The histological scoring for NAFLD was conducted as determined by the criteria of the Pathology Committee of the Nonalcoholic Steatohepatitis Clinical Research Network (25).

RT-PCR and mRNA analysis. Total RNA was extracted from liver tissues using TRIzol reagent (Invitrogen Life Technologies, Carlsbad, CA, USA) and quantified using a NanoDrop 1000 spectrophotometer (Thermo Fisher Scientific, Waltham, MA, USA). cDNA synthesis was performed using $2 \mu \mathrm{g}$ total RNA and Moloney murine leukemia virus reverse transcriptase with oligo dT primers (Fermentas, Burlington, ON, Canada) or an miRNA RT primer set (Guangzhou RiboBio Co., Ltd., Guangzhou, China). RT-PCR was performed on an ABI 7500 PCR system (Applied Biosystems, Foster City, CA, USA) using an SYBR-Green PCR kit (Beijing CoWin Biotech Co.,Ltd., Beijng, China). The expression levels of the miRNAs were normalized to those of U6 small nuclear RNA and the expression levels of the target genes were normalized to those of $\beta$-actin. The relative expression level of each miRNA and target gene was assessed by the $2^{-\Delta \Delta \mathrm{Ct}}$ method (26). All RT-PCR reactions were conducted in triplicate. The primers used were as follows: NCOR1 forward: 5'-CCCATTTCCAGCGTGTTAGTG-3' and reverse: 5'-AAGGTGAAGCAT TTTGGTCATCT-3'; $\beta$-actin forward: 5'-GAGACCTTCAACACCCCAGC-3' and reverse: 5'-ATGTCACGCACGATTTCCC-3'.

Bioinformatic analysis. As determined by a previous study (27) and the Gene Expression Omnibus, miR-199a-5p was selected for computational prediction using TargetScanMouse v6.2 (www.targetscan.org) to generate a list of target genes. For functional analysis of miR-199a-5p targets, an enrichment analysis of the predicted target genes was performed using the Database for Annotation, Visualization and Interrogated Discovery (DAVID) (28). The enrichment analysis was conducted with all known gene ontology (GO) terms and Kyoto Encyclopedia of Genes and Genomes (KEGG; www.genome.jp/kegg) pathways.

Western blotting. Tissue samples were extracted using radioimmunoprecipitation assay buffer containing $1 \%$ phenylmethanesulfonyl fluoride, and protein concentrations were measured by the Bradford method (Bio-Rad, Hercules, CA, USA). Samples of equal protein quantities (50 $\mu \mathrm{g} /$ well) were separated using SDS-PAGE and transferred to polyvinylidene difluoride membranes (Amersham PLC, Amersham, UK) by electroblotting. The membranes were incubated overnight at $4^{\circ} \mathrm{C}$ with primary rabbit antibodies against NCOR1 [diluted 1:1,000 in phosphate-buffered saline (PBS); Cell Signaling Technology, Inc., Danvers, MA, USA], transforming growth factor (TGF)- $\beta 1$ (diluted 1:1,000 in PBS; Abcam, Cambridge, 

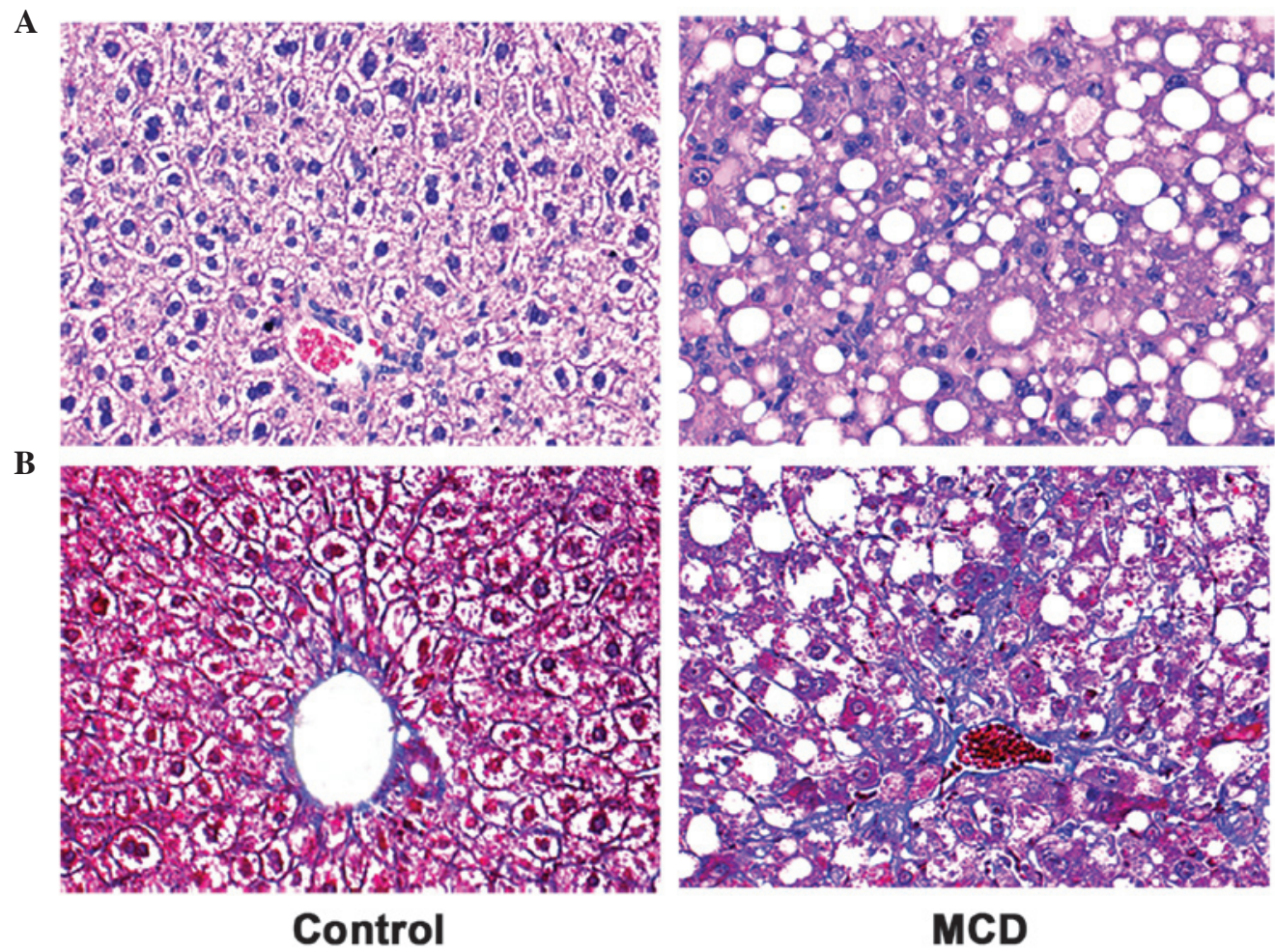

MCD

Figure 1. Liver sections of C57BL/6J mice fed control and MCD diets for eight weeks. (A) Hematoxylin and eosin staining; (B) Masson's trichrome staining. Original magnification, $\mathrm{x} 200 . \mathrm{MCD}$, methionine-choline-deficient.

UK) or $\alpha$-smooth muscle actin ( $\alpha$-SMA; diluted 1:1,000 in PBS; Abcam). Signals were normalized to those of $\beta$-actin, detected by antibodies purchased from Sigma-Aldrich (diluted 1:1,000 in PBS; St. Louis, MO, USA). Following incubation with goat-anti-rabbit IRDye800 secondary antibody (Rockland, Gilbertsville, PA, USA), the bands detected on the membranes were quantified using an Odyssey ${ }^{\mathrm{TM}}$ Infrared Imager (LI-COR Biosciences, Inc., Lincoln, NE, USA).

Cell culture and transfection. LX-2 HSCs were purchased from American Type Culture Collection (Rockville, MD, USA). HSCs are a major fibrogenic cell type that contribute to collagen accumulation during chronic liver disease and constitute valuable tools in the analysis of liver diseases. LX-2 cells were cultured in Dulbecco's modified Eagle's medium supplemented with 10\% fetal bovine serum, $100 \mathrm{U} / \mathrm{ml}$ penicillin and $100 \mathrm{~g} / \mathrm{l}$ streptomycin at $37^{\circ} \mathrm{C}$ in a humidified environment with $5 \% \mathrm{CO}_{2}$. The cells were seeded at a density of $2 \times 10^{5}$ cells $/ \mathrm{ml}$ and grown to $50 \%$ confluency prior to transfection with $100 \mathrm{nmol} / \mathrm{l} \mathrm{miR}-199 \mathrm{a}-5 \mathrm{p}$ mimic, miR-199a-5p inhibitor or the corresponding controls (Guangzhou RiboBio Co., Ltd.) using Lipofectamine ${ }^{\circledR} 2000$ (Invitrogen Life Technologies). The transfected cells were cultured for $48 \mathrm{~h}$ and harvested for total RNA and protein extraction. The samples were stored at $-80^{\circ} \mathrm{C}$ for subsequent use in RT-PCR or western blotting assays. Each treatment was conducted in at least three replicates.

Statistical analysis. All results are presented as mean \pm standard deviation. The data were analyzed using independent Student's t-test or one-way analysis of variance with SPSS 16.0 software (29). $\mathrm{P}<0.05$ was considered to indicate a statistically significant difference.

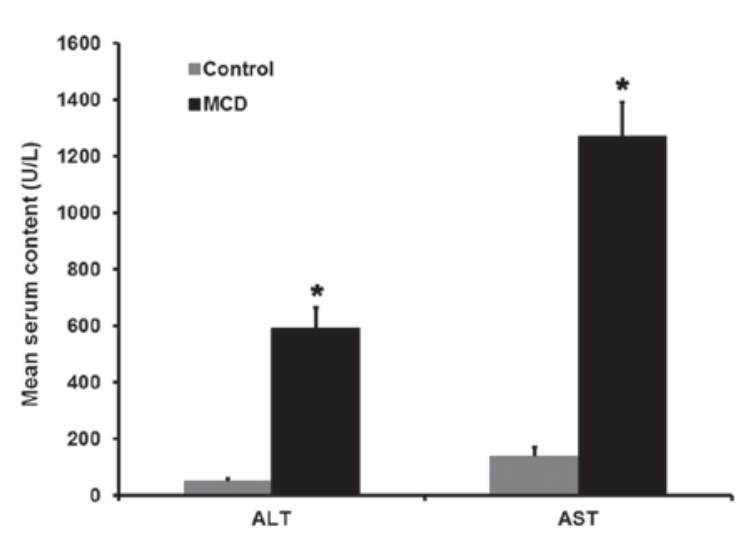

Figure 2. Serum transaminase levels in $\mathrm{C} 57 \mathrm{BL} / 6 \mathrm{~J}$ mice fed either a control or an MCD diet for eight weeks. $\mathrm{N}=6$. ${ }^{*} \mathrm{P}<0.05$ vs. control, t-test. MCD, methionine-choline-deficient; ALT, alanine aminotransferase; AST, asparate aminotransferase.

\section{Results}

Hepatic inflammation and fibrosis following the MCD diet. To develop an animal model of NASH, mice were fed a MCD diet for eight weeks (4,5). MCD-treated animals exhibited disordered lobule structure, severe macrosteatosis, focal hepatocyte necrosis, inflammatory infiltration, portal fibrosis and fibrous septa (Fig. 1). Furthermore, the MCD diet significantly increased the expression levels of the serum transaminases, ALT and AST ( $\mathrm{P}<0.05$; Fig. 2), indicating that the diet induced the characteristic steatosis pathology, mixed hepatic inflammatory and fibrosis, signifying a successful NASH model. 
Table I. Functional analysis of miR-199a-5p targets.

miR-199a-5p targets found by GO-term analysis P-value

Biological process

GO:0045449 - regulation of transcription

GO:0010604 - positive regulation of

macromolecule metabolic process

GO:0006357 - regulation of transcription

from RNA polymerase II promoter

GO:0045941 - positive regulation of

transcription

GO:0010628 - positive regulation of

gene expression

Cellular component

GO:0044459 - plasma membrane part

$1.50 \times 10^{-3}$

GO:0030054 - cell junction

$5.71 \times 10^{-3}$

GO:0000267 - cell fraction

$8.51 \times 10^{-3}$

GO:0031988 - membrane-bounded vesicle

$3.17 \times 10^{-2}$

GO:0005667 - transcription factor complex

$4.41 \times 10^{-2}$

Molecular function

GO:0004674 - protein serine/threonine

$1.14 \times 10^{-4}$

kinase activity

GO:0008375 - acetylglucosaminyltransferase $\quad 2.59 \times 10^{-4}$

activity

GO:0003712 - transcription cofactor activity

GO:0008134 - transcription factor binding

$1.06 \times 10^{-3}$

$1.37 \times 10^{-3}$

$3.46 \times 10^{-2}$
Table II. Pathways associated with miR-199a-5p identified by KEGG analysis.

\begin{tabular}{lcc}
\hline Pathway & P-value & $\begin{array}{c}\text { Predicted targets } \\
\text { in pathway }\end{array}$ \\
\hline Insulin signaling pathway & 0.008 & 8 \\
Regulation of actin cytoskeleton & 0.010 & 10 \\
Wnt signaling pathway & 0.012 & 8 \\
MAPK signaling pathway & 0.013 & 11 \\
Adherens junction & 0.038 & 5 \\
\hline
\end{tabular}

miR, microRNA; KEGG, Kyoto Encyclopedia of Genes and Genomes; MAPK, mitogen-activated protein kinase.

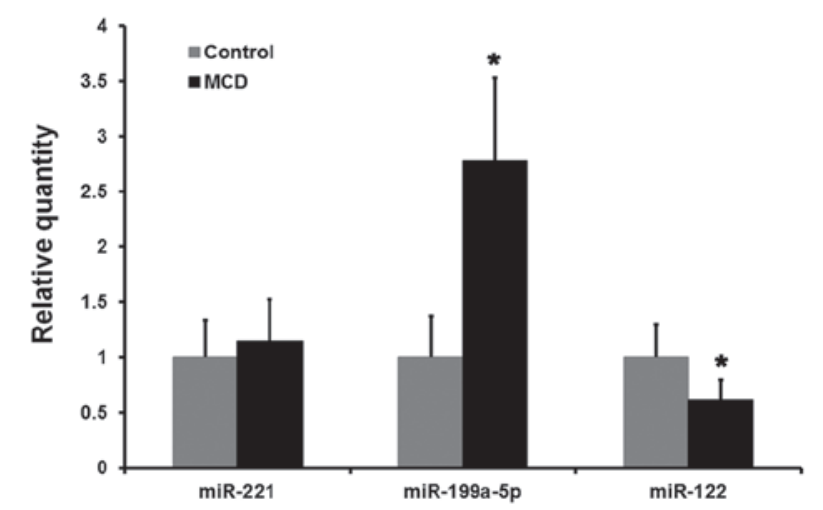

Figure 3. Effects of MCD diet on hepatic miR-221, miR-199a-5p and miR-122 expression levels. Data are presented as the average fold-change relative to the control diet. Experiments were repeated three times $(n=6) .{ }^{*} \mathrm{P}<0.05$ vs. control, t-test. MCD, methionine-choline-deficient; miR, microRNA.
Dysregulation of miRNAs in the livers collected from C57BL/6J mice fed an MCD diet. Using microarray experiments, Pogribny et al (19) demonstrated that feeding C57BL/6J mice a methyl-deficient diet to induce NASH results in aberrant expression of miRNAs, including miR-122, miR-199a-5p and miR-221. To investigate the role of these three miRNAs in $\mathrm{NASH}$, the levels of miR-122, miR-199a-5p and miR-221 were determined in the livers extracted from mice fed MCD and control diets, using RT-PCR experiments. The MCD diet was found to significantly increase miR-199a-5p expression levels and significantly reduce miR-122 expression levels in rat livers ( $\mathrm{P}<0.05$; Fig. 3).

Functional analysis of miR-199a-5p target genes. A single miRNA may regulate numerous target protein-coding mRNAs at the posttranscriptional level. Therefore, the alteration of multiple biological functions may be associated with miRNA dysregulation. To understand the functions of a particular miRNA, identification of corresponding target genes is an indispensable step. miR-122, the most abundant liver miRNA, is implicated in altered lipid metabolism and therefore NASH pathogenesis (20). NASH was associated with altered hepatic miRNA expression (20). However, the role of miR-199a-5p in NASH etiology has not been elucidated. Therefore, in the present study, miR-199a-5p target genes were identified using Target Scan Mouse v6.2 and enrichment analysis was performed on predicted target genes employing DAVID. The results obtained from the GO-term analysis revealed an association between miR-199a-5p and the regulation of certain processes, including transcription, transcription factor complex and protein serine/threonine kinase activity (Table I). KEGG analysis revealed the involvement of miR-199a-5p in a variety of pathways, including insulin signaling, and the Wnt and mitogen-activated protein kinase (MAPK) signaling pathways (Table II). In conclusion, the findings suggest a role of miR-199a-5p in the regulation of NASH.

miR-199a-5p targets gene expression at the protein level following the MCD diet. To determine the exact target genes of miR-199a-5p, the three most common prediction algorithms, TargetScan 6.2, miRanda and PITA were employed. The target genes identified by all algorithms were further evaluated. NCOR1, one predicted target gene, was reported to be required for the integration of inflammatory and anti-inflammatory signals that are associated with the pathogenesis of NASH (30). As shown in Fig. 4, increased hepatic miR-199a-5p expression levels following administration of a MCD diet are associated 
A

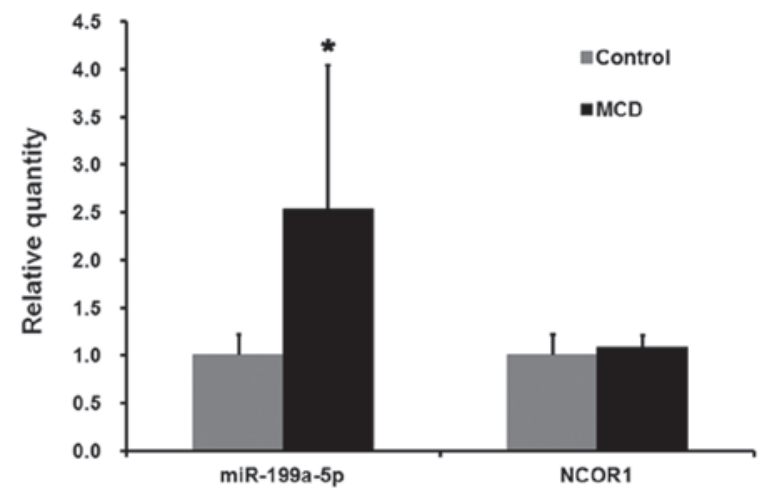

B

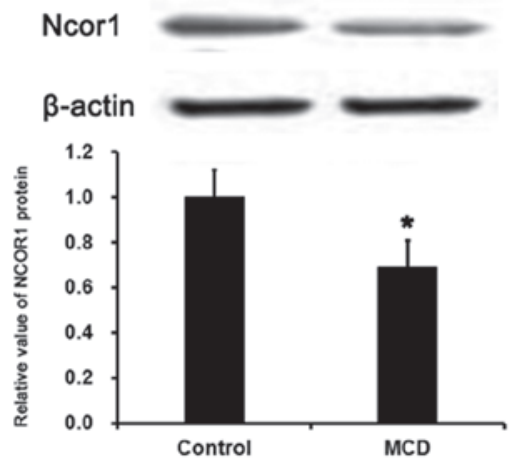

Figure 4. miR-199a-5p and NCOR1 expression level changes in the livers of C57BL/6J mice fed control or MCD diets for eight weeks. (A) miR-199a-5p and NCOR1 mRNA expression levels evaluated by reverse transcription polymerase chain reaction. (B) NCOR1 protein expression levels as assessed by western blotting. Experiments were performed three times, independently $(\mathrm{n}=6){ }^{*} \mathrm{P}<0.05$ vs. controls, t-test. miR, microRNA; NCOR1, nuclear receptor corepressor 1 ; MCD, methionine-choline-deficient.

A

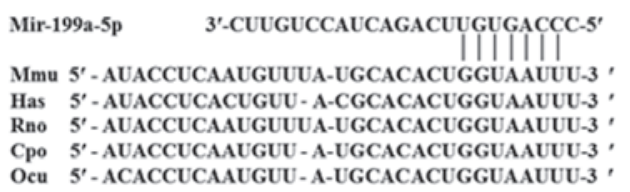

D

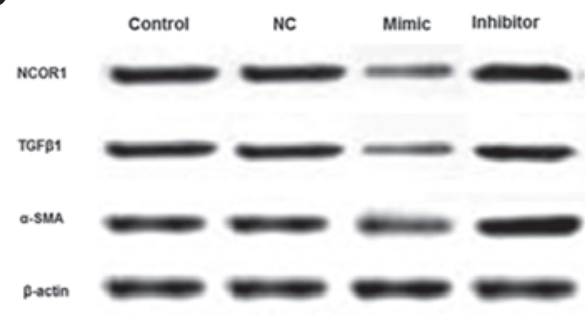

B

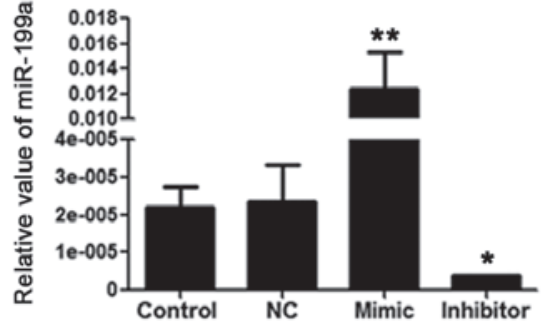

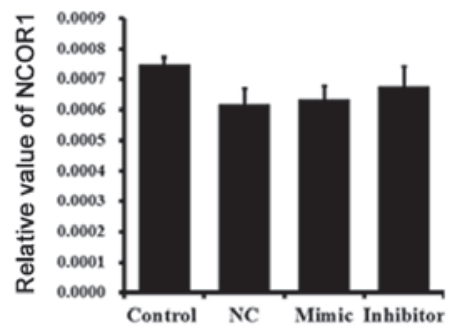

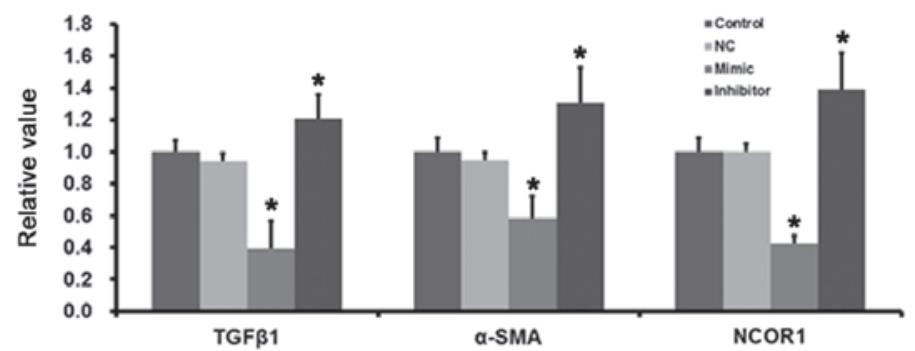

Figure 5. miR-199a-5p negatively regulates the expression levels of NCOR1 protein in LX-2 human hepatic stellate cells. (A) Conservation analysis of predicted miR-199a-5p binding sites in the NCOR1 3'-untranslated region across different species. (B) RT-PCR analysis of miR-199a-5p in LX-2 cells following transfection with miR-199a-5p mimic, inhibitor or NC. (C) RT-PCR quantification of NCOR1 mRNA expression levels. (D) Western blot analysis of NCOR1, TGF- $\beta 1$ and $\alpha$-SMA protein expression levels following transfection with miR-199a-5p mimic, inhibitor or $\mathrm{NC}(\mathrm{n}=6) .{ }^{*} \mathrm{P}<0.05,{ }^{* *} \mathrm{P}<0.01$ vs. NC, one-way analysis of variance. miR, microRNA; NCOR1, nuclear receptor corepressor 1; RT-PCR, reverse transcription polymerase chain reaction; NC, negative control; TGF, transforming growth factor; $\alpha$-SMA, $\alpha$-smooth muscle actin.

with reduced NCOR1 expression at the protein, but not the mRNA level.

Correlation between miR-199a-5p and NCOR1 expression levels in LX-2 cells. To further investigate whether miR-199a-5p regulates NCOR1 translation, either $100 \mathrm{nmol} / \mathrm{l}$ miR-199a-5p mimic, inhibitor or a negative control (NC) were transfected into LX-2 HSCs, respectively. NCOR1 mRNA expression levels were not affected by miR-199a-5p mimic, inhibitor or NC transfection, although NCOR1 protein expression levels were significantly reduced by miR-199a-5p overexpression and significantly increased by miR-199a-5p knockdown $(\mathrm{P}<0.05)$. In addition, miR-199a-5p expression levels were inversely correlated with the expression levels of several fibrosis-associated genes, including TGF- $\beta$ and $\alpha$-SMA (Fig. 5).

\section{Discussion}

Post-transcriptional regulation of miRNAs is widely acknowledged as an important contributor to the pathogenesis of numerous diseases, including NASH. Prior studies have observed that altered miRNAs are involved in insulin signal transduction, inflammatory responses, and lipid and glucose homeostasis in NASH (31-33). However, the underlying mechanisms of the pathophysiological regulation are not fully understood. In the present study, C57BL/6J mice were fed an MCD diet to develop a form of fibrosing steatohepatitis in order to identify the role of altered miRNAs in the NASH pathological process. Histological analysis revealed that the MCD diet disrupted the hepatic lobule structure and resulted in severe macrosteatosis, focal hepatocyte necrosis and inflammatory infiltration, as well as significant elevation of serum ALT and 
AST levels. In addition, a significant reduction in miR-122 expression levels and significant upregulation of miR-199a-5p expression were observed in the NASH mouse model. These data indicate that dysregulated expression of miR-122 and miR-199a-5p may be critical in NASH pathogenesis.

The pathophysiology of NASH is associated with signs of the metabolic syndrome, including disordered lipid metabolism. According to the two-hit hypothesis, increased intrahepatic triglyceride accumulation may result in NASH. miR-122, the most abundant miRNA in the liver, has been reported to mainly affect lipid metabolism by regulating the expression of SREBP-1c, Fas and HMG-CoA (34-36). Indeed, downregulation of miR-122 was observed in the livers of ob/ob mice, a well-established hyperglycemic model of NAFLD, and in human NASH patients $(20,37)$. Thus, miR-122, a key regulator of cholesterol and fatty-acid metabolism, may be an attractive therapeutic target for metabolic diseases, including NASH. miR-199 has been reported to affect the proliferation and apoptosis of hepatoma carcinoma cells (38), and regulates the TGF- $\beta$ signaling pathway, by directly targeting Smad4 (39). Furthermore, Murakami et al (16) have shown that overexpression of miR-199 is associated with the progression of liver fibrosis. However, the pivotal role of miR-199a-5p in the pathogenesis of NASH is not fully understood.

In the present study, to detect the potential role of dysregulated miR-199a-5p in the pathogenesis of NASH, bioinformatic analysis of miR-199a-5p target genes was performed using DAVID (30). The majority of the pathways predicted to be affected by miR-199a-5p were found to be associated with processes involved in the progression of liver fibrosis, such as insulin signaling, regulation of actin cytoskeleton, Wnt signaling, MAPK signaling and adherens junctions. Further analysis using three algorithms (TargetScan, miRanda and PITA) (40) indicated that NCOR1 is a potential target of miR-199a-5p. To investigate this possibility, the expression levels of NCOR1 were analyzed by miR-199a-5p overexpression or knockdown in LX-2 HSC cells. The in vitro experiments confirmed that miR-199a-5p expression levels were inversely correlated with the expression levels of NCOR1.

By producing extracellular matrix upon injury, HSCs are important in the pathological progression of NASH-related fibrosis (41). Under normal conditions, HSCs reside in the space of Disse and store vitamin A in lipid droplets. Upon chronic injury, HSCs undergo marked phenotypic activation and transdifferentiate into myofibroblast cells with the acquisition of contractile, proinflammatory and fibrogenic properties (42-44). Peroxisome proliferator-activated receptor gamma (PPAR- $\gamma$ ), a nuclear transcription factor, is essential in inhibiting HSC activation and proliferation $(29,45)$. NCoR is an adaptor protein that promotes cyclin-dependent 5-mediated PPAR $\gamma$ Ser-273 phosphorylation and transrepresses the activation of PPAR $\gamma$, therefore, downregulation of $\mathrm{NCoR}$ maintains HSCs in a quiescent state (46). Cooperative NCoR/silencing mediator of retinoic acid and thyroid hormone receptor interaction is also required for the integration of inflammatory and anti-inflammatory signals, thus may affect the development of NASH via inflammation (47). Furthermore, the effects of miR-199a-5p expression in LX-2 cells on the following key proteins: TGF- $\beta 1$, which regulates hepatic fibrosis, and $\alpha$-SMA, which is involved in matrix remodeling, were evaluated in the present study. Therefore, overexpression of miR-199a-5p may contribute to the development of NASH by regulating NCOR1 at the posttranscriptional level. These data suggest that miR-199a-5p and NCOR1 may be molecularly targeted for the modulation of NASH.

In conclusion, the present study demonstrated that miR-199a-5p regulates the expression of NCOR1 by inhibiting the translation of NCOR1 mRNA. These findings suggest that abnormal expression of miR-199a-5p may contribute to the pathogenesis of NASH, at least in part, by affecting NCOR1 expression. This provides novel insights into the mechanisms of NASH pathogenesis. However, further studies of human $\mathrm{NASH}$ are required to verify the role of miR-199a-5p in NASH etiopathology.

\section{Acknowledgements}

This study was supported by Hebei Provincial Natural Science Fund (grant no. H2013206276).

\section{References}

1. Adams LA, Lymp JF, St Sauver J, et al: The natural history of nonalcoholic fatty liver disease: a population-based cohort study. Gastroenterology 129: 113-121, 2005.

2. Farrell GC and Larter CZ: Nonalcoholic fatty liver disease: from steatosis to cirrhosis. Hepatology 43 (2 Suppl 1): S99-S112, 2006.

3. Marra F, Gastaldelli A, Svegliati Baroni G, Tell G and Tiribelli C: Molecular basis and mechanisms of progression of non-alcoholic steatohepatitis. Trends Mol Med 14: 72-81, 2008.

4. Zhang BH, Weltman M and Farrell GC: Does steatohepatitis impair liver regeneration? A study in a dietary model of non-alcoholic steatohepatitis in rats. J Gastroenterol Hepatol 14: 133-137, 1999.

5. Rinella ME, Elias MS, Smolak RR, et al: Mechanisms of hepatic steatosis in mice fed a lipogenic methionine choline-deficient diet. J Lipid Res 49: 1068-1076, 2008.

6. Sutti S, Jindal A, Locatelli I, et al: Adaptive immune responses triggered by oxidative stress contribute to hepatic inflammation in NASH. Hepatology 59: 886-897, 2014.

7. Pogribny IP and Beland FA: Role of microRNAs in the regulation of drug metabolism and disposition genes in diabetes and liver disease. Expert Opin Drug Metab Toxicol 9: 713-724, 2013.

8. Ambros V: The functions of animal microRNAs. Nature 431: 350-355, 2004.

9. Lewis BP, Burge CB and Bartel DP: Conserved seed pairing, often flanked by adenosines, indicates that thousands of human genes are microRNA targets. Cell 120: 15-20, 2005.

10. Bartel DP: MicroRNAs: genomics, biogenesis, mechanism, and function. Cell 116: 281-297, 2004.

11. Bartel DP: MicroRNAs: target recognition and regulatory functions. Cell 136: 215-233, 2009.

12. Najafi-Shoushtari SH, Kristo F, Li Y, et al: MicroRNA-33 and the SREBP host genes cooperate to control cholesterol homeostasis. Science 328: 1566-1569, 2010.

13. El Ouaamari A, Baroukh N, Martens GA, et al: miR-375 targets 3'-phosphoinositide-dependent protein kinase-1 and regulates glucose-induced biological responses in pancreatic beta-cells. Diabetes 57: 2708-2717, 2008.

14. Shan Y, Zheng J, Lambrecht RW and Bonkovsky HL: Reciprocal effects of micro-RNA-122 on expression of heme oxygenase-1 and hepatitis $\mathrm{C}$ virus genes in human hepatocytes. Gastroenterology 133: 1166-1174, 2007.

15. Ogawa T, Enomoto M, Fujii H, et al: MicroRNA-221/222 upregulation indicates the activation of stellate cells and the progression of liver fibrosis. Gut 61: 1600-1609, 2012.

16. Murakami Y, Toyoda H, Tanaka M, et al: The progression of liver fibrosis is related with overexpression of the miR-199 and 200 families. PLoS One 6: e16081, 2011.

17. Murakami Y, Toyoda H, Tanahashi $\mathrm{T}$, et al: Comprehensive miRNA expression analysis in peripheral blood can diagnose liver disease. PLoS One 7: e48366, 2012. 
18. Zhang H, Li QY, Guo ZZ, et al: Serum levels of microRNAs can specifically predict liver injury of chronic hepatitis B. World J Gastroenterol 18: 5188-5196, 2012.

19. Pogribny IP, Starlard-Davenport A, Tryndyak VP, et al: Difference in expression of hepatic microRNAs miR-29c, miR-34a, miR-155, and miR-200b is associated with strain-specific susceptibility to dietary nonalcoholic steatohepatitis in mice. Lab Invest 90 : 1437-1446, 2010

20. Cheung O, Puri P, Eicken C, et al: Nonalcoholic steatohepatitis is associated with altered hepatic MicroRNA expression. Hepatology 48: 1810-1820, 2008.

21. Alenghat T, Meyers K, Mullican SE, et al: Nuclear receptor corepressor and histone deacetylase 3 govern circadian metabolic physiology. Nature 456: 997-1000, 2008.

22. Fozzatti L, Park JW, Zhao L, Willingham MC and Cheng SY: Oncogenic Actions of the Nuclear Receptor Corepressor (NCOR1) in a Mouse Model of Thyroid Cancer. PLoS One 8: e67954, 2013.

23. Nan YM, Han F, Kong LB, et al: Adenovirus-mediated peroxisome proliferator activated receptor gamma overexpression prevents nutritional fibrotic steatohepatitis in mice. Scand J Gastroenterol 46: 358-369, 2011.

24. Jian-gao F; Chinese Liver Disease Association: Guidelines for management of nonalcoholic fatty liver disease: an updated and revised edition. Zhonghua Gan Zang Bing Za Zhi 18: 163-166, 2010

25. Kleiner DE, Brunt EM, Van Natta M, et al; Nonalcoholic Steatohepatitis Clinical Research Network: Design and validation of a histological scoring system for nonalcoholic fatty liver disease. Hepatology 41: 1313-1321, 2005.

26. Livak KJ and Schmittgen TD: Analysis of Relative Gene Expression Data using Real-Time Quantitative PCR and the 2(-Delta Delta C(T)) Method. Methods 25: 402-408, 2001.

27. Cheung HH, Davis AJ, Lee TL, Pang AL, Nagrani S, Rennert OM and Chan WY. Methylation of an intronic region regulates miR-199a in testicular tumor malignancy. Oncogene 30: 3404-3415, 2011

28. Huang da W, Sherman BT and Lempicki RA: Systematic and integrative analysis of large gene lists using DAVID bioinformatics resources. Nat Protoc 4: 44-57, 2009.

29. Galli A, Crabb DW, Ceni E, et al: Antidiabetic thiazolidinediones inhibit collagen synthesis and hepatic stellate cell activation in vivo and in vitro. Gastroenterology 122: 1924-1940, 2002.

30. Huang da W, Sherman BT and Lempicki RA: Bioinformatics enrichment tools: paths toward the comprehensive functional analysis of large gene lists. Nucleic Acids Res 37: 1-13, 2009.

31. Rottiers V and Näär AM: MicroRNAs in metabolism and metabolic disorders. Nat Rev Mol Cell Biol 13: 239-250, 2012.

32. Dávalos A, Goedeke L, Smibert $\mathrm{P}$, et al: miR-33a/b contribute to the regulation of fatty acid metabolism and insulin signaling. Proc Natl Acad Sci USA 108: 9232-9237, 2011.
33. Poy MN, Eliasson L, Krutzfeldt J, et al: A pancreatic islet-specific microRNA regulates insulin secretion. Nature 432: 226-230, 2004.

34. Esau C, Davis S, Murray SF, et al: miR-122 regulation of lipid metabolism revealed by in vivo antisense targeting. Cell Metab 3 : 87-98, 2006.

35. Chang J, Nicolas E, Marks D, et al: miR-122, a mammalian liver-specific microRNA, is processed from hor mRNA and may downregulate the high affinity cationic amino acid transporter CAT-1. RNA Biol 1: 106-113, 2004.

36. Krützfeldt J, Rajewsky N,Braich R, et al: Silencing of microRNAs in vivo with 'antagomirs'. Nature 438: 685-689, 2005.

37. Li S, Chen X, Zhang H, et al: Differential expression of microRNAs in mouse liver under aberrant energy metabolic status. J Lipid Res 50: 1756-1765, 2009.

38. Xu N, Zhang J, Shen C, et al: Cisplatin-induced downregulation of miR-199a-5p increases drug resistance by activating autophagy in HCC cell. Biochem Biophys Res Commun 423: 826-831, 2012

39. Zhang Y, Fan KJ, Sun Q, et al: Functional screening for miRNAs targeting Smad4 identified miR-199a as a negative regulator of TGF-beta signalling pathway. Nucleic Acids Res 40: 9286-9297, 2012.

40. Masotti A and Alisi A: Integrated bioinformatics analysis of microRNA expression profiles for an in-depth understanding of pathogenic mechanisms in non-alcoholic fatty liver disease. J Gastroenterol Hepatol 27: 187-188, 2012.

41. Gäbele E, Brenner DA and Rippe RA: Liver fibrosis: signals leading to the amplification of the fibrogenic hepatic stellate cell. Front Biosci 8: d69-d77, 2003.

42. Geerts A: History, heterogeneity, developmental biology, and functions of quiescent hepatic stellate cells. Semin Liver Dis 21: 311-335, 2001.

43. Milani S, Herbst H, Schuppan D, et al: Procollagen expression by nonparenchymal rat liver cells in experimental biliary fibrosis Gastroenterology 98: 175-184, 1990.

44. Marra F: Hepatic stellate cells and the regulation of liver inflammation. J Hepatol 31: 1120-1130, 1999.

45. Marra F, Efsen E, Romanelli RG, et al: Ligands of peroxisome proliferator-activated receptor gamma modulate profibrogenic and proinflammatory actions in hepatic stellate cells. Gastroenterology 119: 466-478, 2000.

46. Li P, Fan W, Xu J, et al: Adipocyte NCoR knockout decreases PPARgamma phosphorylation and enhances PPARgamma activity and insulin sensitivity. Cell 147: 815-826, 2011.

47. Ghisletti S, Huang W, Jepsen K, et al: Cooperative NCoR/SMRT interactions establish a corepressor-based strategy for integration of inflammatory and anti-inflammatory signaling pathways. Genes Dev 23: 681-693, 2009. 